\title{
The Application of Two Different Types of Context in the Teaching of an Undergraduate Course
}

\author{
Yan Wu \\ Department of Library and Information Studies, The University of West Indies, Kingston, Jamaica \\ Email: yan.wu@uwimona.edu.jm
}

Received 20 October 2015; accepted 16 February 2016; published 23 February 2016

\begin{abstract}
We address the question of how natural learning context and application of visual materials can positively affect the student learning. After discussing theory, we report an application of natural learning context and application of visual materials in the teaching of a recent undergraduate preservation course. In this study, the research questions addressed are: 1) What are the differences between a natural learning situation and a conventionally designed learning situation? 2) How does this difference affect a learner? 3) Why the follow-up activities and application of visual resources are beneficial? This study also points out the function of emotions in cognitive process and adopts the view that all representations arise with the contingent of a context in which emotions are implied, activated, and experienced.
\end{abstract}

\section{Keywords}

\section{Context, Natural Learning Situation, Visual Resources}

\section{Introduction}

Preservation is a recent hot topic in the programs offered by library and information science studies. Although preservation of library books is historically a formal part of library subject, it was not being incorporated in the mainstream of the program's curricula. With the development of the information industry due to the fast advance of technology and a rising concern about materials in digital format, preservation has been offered as a course by an increasing number of LIS programs in the higher institutions. Issues related to preservation are often culturally and historically related; therefore teaching the course usually faces challenges of how to introduce the content to the students.

This paper reports the activities applied to the recent teaching of a preservation class and describes the students' cognitive processes and learning effects associated with such activities and implied learning contexts. The functions of these learning contexts are also explained.

\subsection{Function of Context}

The function of context has been discussed generously in the literature. Halliday [1] says that context is "fore- 
grounding," "a kind of environmentally motivated prominence," in which certain sets of options are favored, and the meaning of a text resides (p. 149). Context does not only activate memories and internal cognitive structures of the individual, but also facilitate the building of new internal cognitive structures. The necessity of context is therefore incontestable in learning, especially for someone learning something new. Most representational texts have multiple meanings; they are only interpretable in the embodied cognition when the individual actively interacts with the physical world.

In another interpretation, context provides two forms of affordance for cognition: one is non-cognitive affordance, functioning as mere markers to distinguish events and to bring humans to consciousness; the other is cognitive affordance, which allows one to build a structure-like event model for future actions [2]. Non-cognitive affordance shares a similar function with emotion in representation, whereas cognitive affordance is referential. This also means that, to provide a rich context to the individual, it also allows emotion to participate in the perceptual process and to facilitate thinking and memory by coupling events with feelings. As such, learning the content also includes learning the regulatory component-emotion - that associates with it [3].

Two different types of context exist. The first is the context of the social and physical world; the other is the dialect relationship between text and context. In the latter case, "the text creates the context as much as the context creates the text," which includes both the intertextual and intratextual contexts ([4], p. 47). Context allows association. Association is a critical faculty in nearly all cognition. Association makes mental images alive and allows forming of new connections. Association is required when new knowledge is acquired. In addition, an individual cognizes an entity only to the extent that she or he sees it from a unity, and from which she or he can draw part-entirety relative characteristics for the entity [5]. Because of this, it is critical to use rich contexts to enlarge the cognitive domain of the individual, whether they are artifacts from the physical world or systematical abstract entities that function as context to the individual.

In addition, the function of context in representation is also an issue of coherence, whether coherence means the perceptual unity of the real object or the unity of the logics within the text(s). Only when unity is achieved can an internal model over the content be formed. The more connections made over the representational units, the more stable and flexible the model the individual creates, which may benefit later cognition, such as information retrieval.

\subsection{Visual Resources}

The form of the representation indicates its potential level of concreteness, flexibility, and function. Using visual materials to design contextual concepts is more feasible than before. Visual materials bear some distinctive functions. One of the functions comes from the belief that the value of a picture is mainly contextual [6]. Visual representations bring the viewer closer to reality, positively activate mental activities, and facilitate actions [7]-[9]. Therefore, they are experiential, and they encourage the perception-action cycle of cognition [10].

\subsection{Learning Process or Products: Artifacts and Cognition Afforded}

Butterworth [11] contends that, to insist on the contextualization of thought is not enough to explain its nature and development; a proper understanding must explain what motivates problem solving, expressed through individual goals as well as social intentions as defined within the various opportunities provided for the cultural transmission of knowledge. As such, the discussion of representation returns to the individuals' experiential actions afforded by the social contexts in. Again, it is not behaviors themselves that matter but for what the social contexts afford and how they so do.

The emphasis of the context issue in cognition therefore reflects pragmatically in various cognitive interactivities, especially attention, perception, and association when individuals are situated in the context. Participants are actively involved in the detection and utilization of contextual cues, when social artifacts are formed, used and transformed. No perspective, in terms of the distinction between the artifacts and processes from which they are formed in the activities, is more important than the other, as they mirror each other.

\section{Comparison of a Natural Learning Situation and a Classroom Learning Situation}

Learning occurred in the physical world without additional interfaces between the individual and an object of interaction is called a natural learning situation. In contrast, learning in a traditional classroom often has several interfaces involved. These interfaces range from symbolic system with abstract content to artificial platforms 
made by computer software which can affect the motivation of the leaner and further impede the process of learning.

Motivation of cognition comes from a sense of purpose. Based on unconscious, motivation only comes from real social situations, in which senses are able to connect with the mind and the spirit. For Panofsky, the necessity of trying to prove a perspective or thought is "the logical consequence of pursuing the questions of cultural relativism and pictorial realism” ([12], p. 11). This means that the utility for learning a concept or a perspective must be found in the real social and physical world. In an environment where the individual cannot see his or her actual needs of learning as a contention between mind and body, motivation is compromised.

Moreover, perception directly affects memory and, as a result, information retrieval. The cognitive activities involved in direct perception and indirect perception are different. The major actions in direct perception are to perceive and to reflect in an infinite environment, whereas the major actions in indirect perception are to decode from a limited abstract content pool and to use limited logics to rationalize. The actions involved within the latter cannot replace the former from which the continuous structures of the perceptual world are perceived, and their differences also affect memory and information retrieval at a later time. In contrast, naturalistic environments make it possible for people "to use structural features and higher order relations," or in other words, intuition. Furthermore, naturalistic settings "influence the retrieval conditions, often stressing the search for higher-order analogs rather than analogs that share superficial features” ([13], p. 329). Piaget [14] also contends that gestalts are irreducible, and they must be used as a totality and cannot be analyzed by their original constituent elements. This nature of perception greatly differentiates direct perception provided by naturalistic settings from indirect perception provided by conventional settings.

The nature of the content knowledge as we historically has made it, as well as the cognitive activities that allow individual to employ, defines the characteristics of the conventional instructional design. The typical instructional materials contain entities that are social rules and the products of these rules. These representations are constructed based on difference rather than inexhaustible naturalistic relations of the physical world. Therefore, instructional materials are severely limited exemplars that stand for optional perspectives, and they are intended to form particular sets of knowledge that can be perceived and reflected in a particular context.

The characteristics of the conventional instructional materials determine that the individual starts with an indirect perception, and the decoding process overlaps with the perceiving process, or the decoding process totally overrides the perceiving process. The different natures between the conventional instructional settings and the natural environments also define the logic that the individual can apply. Induction and deduction are allowed in an abstractly designed environment, but induction, deduction, and adduction are allowed in a natural environment.

As a consequence, the experiences an individual obtains from interacting with the two environments are also different. In a naturalistic setting, the individual is close to the perceptual world and affects, which both have a physical basis. The open resource allows the individual to have a sense of real experience and to become involved in activities such as "pondering these phenomena in all their aspects" as in the inquiry of abduction ([15], p. 6469). Therefore, self is emphasized in the experience. In a conventionally designed situation in which the source is limited, logical reasoning overrides the individual as explicit rules, which also suffices for the sense instead. Also, self and cognition can be separated, and often are, and because of the separation of self and emotion from cognition, the experience becomes less real.

In addition, because the sensual qualities perceived from the interactions are different between the experiences, memories of them also differ. The copied images formed through interactions with nature are more stable, have more sensual qualities, and usually imply or combine emotions, but the copied images formed through interactions with the abstract content contain more concepts than images, and images generated from the latter are also synthetic or vague, which are treated as secondary and easy to fade away. Moreover, the effects that are associated with image formation also differ, in which the process of imaging through multiple senses, such as visual, touch, and smell, from naturalistic settings are more motivating than those image generated from conventional settings. Table 1 summarizes the differences between the two settings.

The purpose of distinguishing the differences between the two types of learning situation is to delineate a portrait showing how representational resources have been applied, especially in formal knowledge transformation. As such, we can see the drawbacks of the past in terms of designing instructional materials as well as the advantages and options we have now with the increased knowledge and accessibility to representational resources beyond linguistics. Then, we can foresee and plan for the future of education and make efforts to shorten the 
Table 1. A comparison between a natural learning situation and a conventionally designed learning situation.

\begin{tabular}{|c|c|c|}
\hline & Natural Environment & Conventional Environment \\
\hline 1. Nature of Resource & indefinite openness & abstract contrasts \\
\hline 2. Structure of Artifact & $\begin{array}{l}\text { micro-object; } \\
\text { micro-macro-object, } \\
\text { micro-macrostructure; }\end{array}$ & $\begin{array}{l}\text { macro-object; } \\
\text { micro-object-macro-object; }\end{array}$ \\
\hline 3. Perception & direct (cognition depends on perceptual world) & $\begin{array}{l}\text { indirect (cognition does not depend on perceptual world, } \\
\text { and logics override perceptual process) }\end{array}$ \\
\hline 4. Embodiment & embodied cognition & disembodied cognition \\
\hline 5. Cognitive Method & induction, deduction, \& abduction & induction \& deduction \\
\hline 6. Handling of Errors & $\begin{array}{l}\text { heuristic constructive; } \\
\text { adoptive and tolerance }\end{array}$ & no solution or limited solution; less tolerance \\
\hline 7. Effect on Memory & $\begin{array}{l}\text { contain more images; } \\
\text { images are more stable and have more } \\
\text { sensual qualities; and images are motivating }\end{array}$ & $\begin{array}{l}\text { contain less images; } \\
\text { images are vague and easy to fade away and with } \\
\text { less sensual qualities; and images are less motivating }\end{array}$ \\
\hline
\end{tabular}

difference between a natural learning situation and a classroom setting by employing the knowledge of how individuals perceive, interpret, and associate, and by providing learning resources close to an indefinite openness, under the new era of semiotics as well as social development.

\section{An Application of Natural Learning Situation in a Preservation Course}

The class of 30 juniors in the undergraduate LIS program in a known public university in Jamaica carried out the field visits of 3 different preservation facilities as part of the learning activities for a preservation course. Each student was asked to report on one of the facility visits by addressing a group of open-ended questions relevant to preservation practices.

\section{1) Nature of Resource}

During the preservation facility visit, students were introduced to the staff, the history of the lab and the overall facility, the physical structure of the facility, the general practices of preservation carried out, and the materials and tools used. Detailed processes of different preservation practices were demonstrated. All the preservation artifacts were open to be observed by the students. The resources of learning were unlimited. The openness was what truly motivated the students. This openness is also reinforced by students' trust and belief that they were in the contexts of what they could learn about several topics for the course. So, they were enthusiastic to write down everything they got from their observation and from interactions with the staff. Certainly, this openness would not be readily available to students if without the gracious acceptance and support by the staff from the facilities. Students felt welcomed during the visits.

2) Structure of Artifact

Many phenomena and ideas were new to the students. Everyone took pictures if they had a cell phone with camera during the trips. It seemed that everything is tangible to the students. The experience from staff was immediately shared, the queries were addressed shortly, and the doubts from the students were cleared readily.

\section{3) Perception}

To see is to believe. This is significant during the whole facility visit. Besides the perseveration materials and physical layout of the buildings, perception was supported by stories and detailed demonstrations from the staff. Pictures were taken by students.

4) Embodiment

Emotions were activated, transferred, shared, amplified, and experienced during and following the visits. The appreciation of knowledge and sharing of it were high. The excitement and joyfulness of the students were apparent.

\section{5) Cognitive Method}

The report essay was a success with beautiful and detailed writing of the students. Questions were asked and new preservation issues and challenges were identified. Many students expended their learning to using additional electronic resources in their understanding and interpreting the issues. 
6) Handling of Errors

Students are voluntarily repeated their facility visit to acquire additional materials and to verify their initial understandings. Some knowledge changes were more evident following the facility visit, where they encountered a wide diversity of preservation products and practices. Students were less defensive but glad that they actually learned a lot within a very short period of time from the visits.

7) Effect on Memory

The students were taking reference from the pictures taken from the facility. They were excited, talking vividly after their visit during time of discussion, and the essay writing is rich in detailed descriptions.

From this experience, we conclude that a naturalistic context engages the individual to interact with the physical and social world in an active and direct perception, by providing objects with all forms of visibility or sensibility. When there is a need or a self-imposed purpose of learning, emotion contributes to cognition. However, only when the individual actively interacts with the indefinite perceptual world and is involved in intuitive exploration, or heuristic construction, can emotion be tightly embodied in cognition. In other words, a natural learning situation allows inquiries at all levels, abduction, induction, and deduction, and activities that lead to different awareness: observing, pondering, classifying, imagining, predicting, inspecting, and verifying from which emotion, motivation, and the individual self and the physical world are embodied. In addition, a natural learning environment has its own method to address errors. This requires that the nature of the resource of the environment be open enough to contain adequate information and mechanisms of problem solving.

\section{Follow-Up Activity and Application of Visual Resources}

The process of learning explored in the study shows how the individual perceives, interprets, and makes use of what has been shown more than taught. The primary sense-making actions are to recognize the tasks through "perceiving" and "decoding" the content using the individual's own frame of reference, prior experiences, feelings, expectations, and priorities, and then, "testing" the interpretations by exerting his or her own representations.

In addition, the engagement with the content should be a recurring process with more than one access to the content. A developing, coherent, and persistent learning process depends on extensive interactions with the content and the identification of new tasks. The design of instructional material therefore should focus on motivating and leading the individuals to involve themselves with the material in a salient, self-directed process with staged learning activities. In order to allow students to come back to the content of the study happened during the facility visits, the students were requested to post the images of preservation activities by using a blog tool provided by an E-learning platform. An image collection was therefore emerged and keywords were created by all students, then additional comments were provided to the images (see Figure 1). This provides an additional support to what students just had learned therefore helps them to reinforce memory.
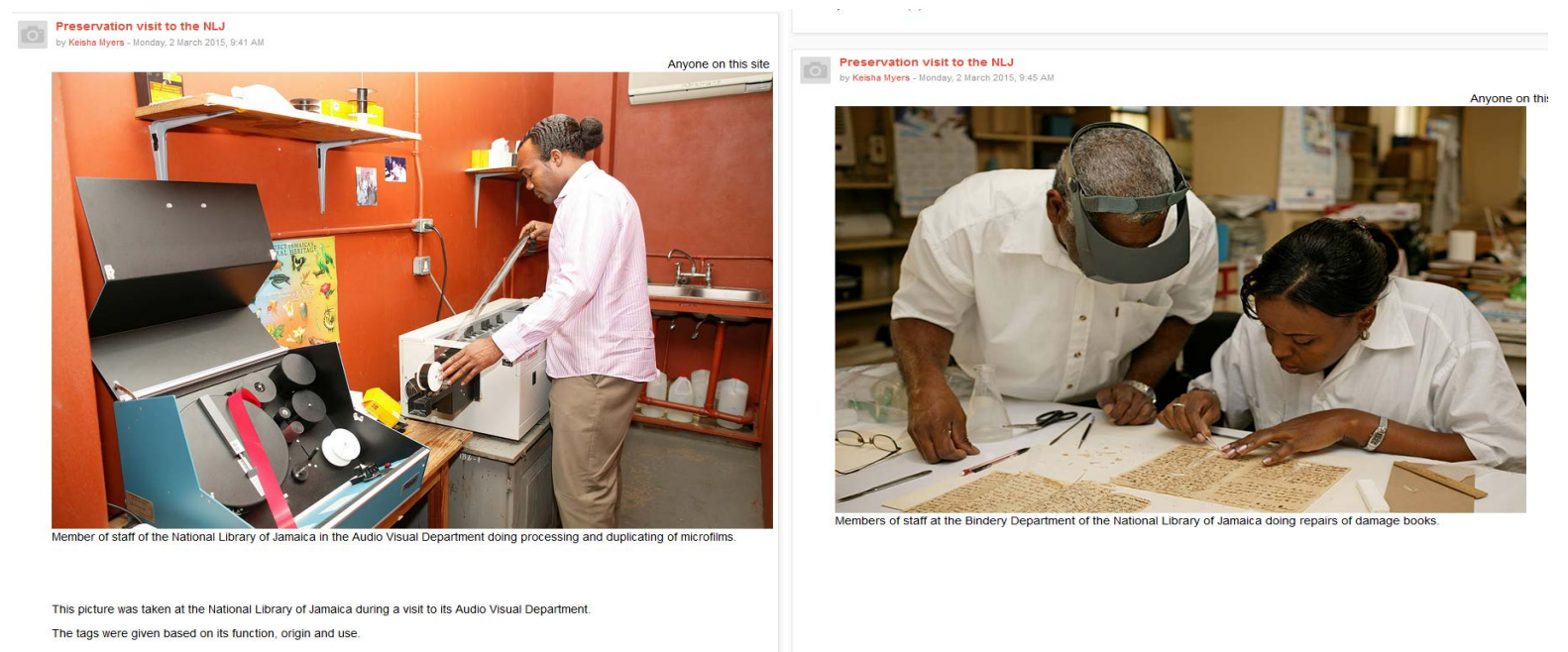

Figure 1. Examples of image blogs. The pictures were taken at the National Library of Jamaica; ourvle.mona.uwi.edu; Online; Nov. 2015. 
So, the image collection and descriptors of images are products of students' active participation in the natural learning context and its follow-up activity back at classroom. The images and keywords as artifacts bear same implications and significance as the processes students went through. They mirror each other in the memory of each student in the study.

\section{Conclusions}

Preservation becomes a hot topic to library studies in the information age. Preservation practice bears cultural sensitivity, often combined with a historical perspective. With such a rich topic, necessary pedagogy was applied in the teaching of the course. Learning, in its various forms, is a process of communication that involves several dimensions of context. For one, if the original context of encoding is not known, such as learning through a traditional classroom, the meaning of the information might not be the same when transferred. Using the reported methods of teaching, we believe the natural learning context strongly affects the way how students learn and interact with the content objects, therefore affects how knowledge was transferred and constructed. The difference starts from perception, and the results come from this perception as well, which include emotion as a component.

One of the implications for contemporary instructional design is to change the natures of the environmental resources or the structures of the artifacts in these resources in a learning situation, in order to provide individuals with more options of cognitive methods and modes and to make the experience of the learning process more coherent and memorable. This implies to adopt those representational methods that transfer a sense of indefinite openness in its external representations. The impacts of these contexts when one interacts with a symbolic system can also be seen therefore through different cognitive processes: discernment, self-regulation, and belief change. If one allows learning to happen in a natural environment, for example, in a field trip, a facility visit, an internship program, these cognitive processes will be carried out easier to students than in classroom settings

\section{References}

[1] Halliday, M.A.K. (1978) Language as a Social Semiotic: The Social Interpretation of Language and Meaning. Edward Arnold, London.

[2] Norman, A.D. (1990) The Design of Everyday Things. Doubleday, New York.

[3] Oatley, K. and Johnson-Laird, P.N. (1995) The Communicative Theory of Emotion: Empirical Tests, Mental Models, and Implications for Social Interaction. In: Martin, L.L. and Tesser, A., Eds., Goals and Affect, Erlbaum, Hillsdale.

[4] Halliday, M.A.K. and Hasan, R. (1985) Language, Context and Text: Aspects of Language in a Social-Semiotic Perspective. Deakin University Press, Geelong.

[5] Arieti, S. (1976) Creativity: The Magic Synthesis. Basic Books, Inc. Publishers, New York.

[6] Hochberg, J. (1987) Machines Should Not See as People Do, but Must Know How People See. Computer Vision, Graphics, and Image Processing, 37, 221-237. http://dx.doi.org/10.1016/S0734-189X(87)80003-8

[7] Ernest, C.H. and Paivio, A. (1969) Imagery Ability in Paired-Associate and Incidental Learning. Psychonomic Science, 15, 181-182. http://dx.doi.org/10.3758/BF03336270

[8] Paivio, A. (1978) On Exploring Visual Knowledge. In: Randhawa, B.S. and Coffman, W.E., Eds., Visual Learning, Thinking, and Communication, Academic Press, New York.

[9] Paivio, A. (1986) Mental Representations: A Dual Coding Approach. Oxford University Press, New York.

[10] Tufte, E.R. (1990) Envisioning Information. Graphics Press, Cheshire.

[11] Butterworth, G. (1993) Context and Cognition in Models of Cognition Growth. In: Light, P. and Butterworth, G., Eds., Context and Cognition: Ways of Learning and Knowing, Lawrence Erlbaum Associates, Publishers, New Jersey.

[12] Elkins, J. (1994) The Poetics of Perspective. Cornell University Press, Ithaca.

[13] Dunbar, K. (2001) The Analogical Paradox: Why Analogy Is So Easy in Naturalistic Settings, Yet So Difficult in the Psychological Laboratory. In: Gentner, D., Holyoak, K.J. and Kokinov, B.N., Eds., The Analogical Mind: Perspectives from Cognitive Science, The MIT Press, Cambridge, 313-334.

[14] Piaget, J. (1952) The Origins of Intelligence in Children. International Universities Press, New York. http://dx.doi.org/10.1037/11494-000

[15] Peirce, C.S. (1960) A Neglected Argument for the Reality of God. In: Hartshorne, C. and Weiss, P., Eds., Collected Papers of Charles Sanders Peirce, Vol. 6, Scientific Metaphysics, Harvard University Press, Cambridge. 